au dictateur Nino Vieira et à l'intervention sénégalaise ressentie par tous comme «péri-française »!

L'ISCSP et l'auteur avaient sans doute des raisons institutionnelles de publier ce mestrado le plus vite possible. Mais on regrettera, sur le plan scientifique, cette précipitation, car on reste sur sa faim relativement au besoin maintenu d'une analyse, sur la durée, du rapport au pouvoir en Guinée-Bissau. Un pas a été fait en ce sens par Fafali Koudawo, Cabo Verde e Guiné-Bissau, da democracia revolucionária à democracia liberal, Bissau, INEP, 2001, 230 p. ("Kacu Martel », 14) et par Lars RÜDEBEGK, en deux versions légèrement différentes, anglaise et portugaise : On Democracy's Sustainability. Transition in Guinea-Bissau, Stockholm, SIDA, 2001, 125 p. («Sida Studies », 4) et Colapso e reconstrução política na Guinée-Bissau, 1998-2000. Um estudo de democratização difícil, Uppsala, Nordiska Afrikainstitutet, 2001, 124 p., ISBN : 91-7106-482-6, livres qui auraient impérativement dû entrer dans la bibliographie de l'auteur.

Souhaitons qu'Álvaro Nóbrega remette main à l'ouvrage, joignant plus profondément histoire, anthropologie politique et sciences politiques.

Mai 2005, Michel Gahen

\title{
Luís Quintais, As guerras coloniais portuguesas e a invenção da História, Lisboune, Imprensa Giências Sociais, novembre 2000, 163 p., ISBN: 972- 671-065-0 ("Estudose Instigações »)
}

Devais-je rédiger cette note de lecture portant sur un domaine qui ne m'est guère familier? En effet, ce livre, publication en novembre 2000 d'une thèse de troisième cycle (mestrado), soutenu en février 1998, se situe avant tout dans le champ de l'anthropologie médicale - même si l'anthropologie des émotions et une étude de la mémoire y ont leur place. Je ne suis donc pas en mesure de commenter la bibliographie et avoue avoir été dépassée par les trois premiers chapitres qui retracent l'histoire des courants de la psychiatrie moderne et reviennent sur l'évolution des définitions du concept de «désordre de stress post-traumatique ». Ma présentation en est, dès le départ, quelque peu biaisée.

Le thème était alléchant: l'auteur revenait sur deux visions différentes, voire antagoniques, de l'histoire: l'une qui réfute toute possibilité de réécriture de l'histoire et l'autre qui pose que toute évocation du passé est systématiquement une réécriture. S'appuyant sur cette dernière, il constate qu'il serait dès lors envisageable de trouver une solution thérapeutique aux maux dont souffrent les vétérans des guerres coloniales, solution permettant une réappropriation du temps et qui se révèlerait efficace y compris sur le plan politique et moral. En utilisant le vocabulaire médical, on inventerait, modèlerait une évocation de cette période historique. Suivant cet objectif, l'auteur participa durant six mois aux séances thérapeutiques du foyer militaire de la Croix Rouge et de l'Association des handicapés des Forces armées (ADFA).

L'auteur se réfère à Afonso de Albuquerque, qu'il cite abondamment et dont le parcours ne peut, selon lui, être dissocié de l'étude des PTSD (post-traumatic stress disorder) au Portugal. Ce psychiatre établit les premiers diagnostics de PTSD en 
1986 suite à une rencontre organisée par l'ADFA sur ce thème où furent invités des spécialistes étrangers. Luís Quintais procède alors à une description des traitements proposés (thérapies individuelles ou de groupe), du corpus (âge, anciens grades ...) et donne une fourchette du nombre d'individus concernés: En juillet 1997, trois cent trente-huit cas étaient diagnostiqués par le Service de psychothérapie comportementale; par ailleurs, à partir des études faites aux E.U.-A. sur les vétérans du Viêt-Nam, Afonso de Albuquerque extrapolait à 140000 hommes le nombre de personnes concernées.

Mais, cette démarche des psychiatres ne reçut pas des anciens combattants un appui unanime. Quelques voix discordantes, comme celle exprimée dans O Combatente (organe de la Ligue des Combattants), plaidèrent, au nom de l'honneur et de l'exaltation que méritent les Combattants, la bonne santé mentale des anciens soldats.

Cela dit, revenant sur les thérapies mises en place et dans le souci de montrer que la mémoire est paradoxalement un acte de création, l'auteur, non sans avoir insisté sur l'idée qu'il faut éviter de faire revire aux anciens combattants les situations douloureuses vécues, explique qu'il s'agit de les faire travailler à la reconstruction de narrations afin qu'ils établissent le lien avec ce qui est tu, oublié, refoulé. Dans ce contexte, à partir de larges extraits des séances auxquelles il a assisté, il se penche sur le processus «d'humanisation de l'inhumain », celui qui pousse des hommes sains à commettre des atrocités puis à souffrir a posteriori de leurs actes.

Dans un dernier chapitre, il souligne les implications non directement thérapeutiques de ce travail de « réécriture rétrospective/narration» des expériences vécues. Selon lui, un lien, une continuité existe entre cet espace de confidences et la scène publique, montrant le chemin parcouru par cette action de consignation des guerres coloniales. De la mémoire individuelle à la mémoire collective, les unités psychiatriques et l'association APOIAR (Association d'appui aux anciens combattants victimes de stress de guerre) deviennent en quelque sorte un «lieu de mémoire».

À l'heure où les sciences sociales commencent à s'intéresser à cette période « oubliée » des guerres coloniales, jusqu'alors évoquée essentiellement à travers la littérature, mes attentes étaient de taille, démesurées. J'espérais de longues analyses sur les liens entre solution/vocabulaire médical et discours politique; je ne pouvais que rester sur ma faim face à un travail ancré dans ce domaine particulier de l'anthropologie médicale. Néanmoins, malgré cet appétit frustré et mon ignorance avérée, le livre me paraît constituer un sérieux outil. La néophyte que je suis est séduite par la qualité de la recherche de Luís Quintais, gourmande que j'étais des témoignages/séances retranscrits et sensible aux fécondes conclusions de cette étude. Ces dernières mériteraient d'ailleurs d'être prises en compte dans d'autres domaines, comme l'histoire notamment, pour des recherches encore trop peu nombreuses sur les Guerres coloniales.

Mai 2005, Judith Manya 